\title{
Transversus Abdominis Release (TAR) Procedure a retrospective analysis of an abdominal wall reconstruction group
}

Carlos Eduardo Rey Chaves ( $\square$ carlosrey991@gmail.com )

Hospital Universitario Mayor Méderi

\section{Felipe Girón}

Universidad del Rosario

Danny Conde

Hospital Universitario Mayor Méderi

Lina Rodriguez

Universidad de Los Andes

David Venegas

Universidad del Rosario

Marco Vanegas

Universidad del Rosario

Manuel Pardo

Universidad del Rosario

Felipe Vargas - Barato

Hospital Universitario Mayor Méderi

Jorge Navarro

Hospital Universitario Mayor Méderi

Alberto Ricaurte

Hospital Universitario Mayor Méderi

\section{Research Article}

Keywords: Component release, Abdominal wall reconstruction, Transversus abdominis release, Herni, Ventral hernia

Posted Date: March 7th, 2022

DOI: https://doi.org/10.21203/rs.3.rs-766601/v2

License: (c) (i) This work is licensed under a Creative Commons Attribution 4.0 International License.

Read Full License 


\section{Abstract \\ Background}

Complex abdominal wall defects are important conditions with a high morbidity, leading to impairment of patients physical condition and quality of life. In the last decade, the abdominal wall reconstruction paradigm has changed due to formation of experienced and excellence groups, improving clinical outcomes after surgery. Therefore, our study shows the perspective and outcomes of an abdominal wall reconstruction group (AWRG) in Colombia, focused on transverse abdominis release (TAR) procedure.

\section{Methods}

A retrospective review of a prospectively collected database was conducted. All the patients older than 18 years old that underwent TAR procedure between January 2014 - December 2020 were included. Analysis and description of postoperative outcomes (recurrence, surgical site infection (SSI), seroma, hematoma, and re-intervention) was performed.

\section{Results}

50 patients underwent TAR procedure. $62 \%$ of patients were male. Mean age was $55 \pm 13.4$ years. Mean BMI was $27.8 \pm 4.5 \mathrm{Kg} / \mathrm{m} 2$. Abdominal wall defects were classified with EHS ventral Hernia classification having a W3 hernia in $72 \%$ of all defects (Mean gap size of $11.49 \mathrm{~cm} \pm 4.03 \mathrm{~cm}$ ). Mean CeDAR preoperative risk score was $20.5 \% \pm 14.5 \%$. Protective association was established for SSI if the procedure was performed by the AWRG OR 0.7 (IC 95\% 0.05-0.93). Higher risk of SSI was found in cases not performed by the abdominal wall reconstruction group OR 13.6 ( $\mathrm{Cl} 95 \% 9.12-15.5)$. In terms of hernia relapse, we have $12 \%$ of cases; all of them over a year after the surgery.

\section{Conclusions}

TAR procedure for complex abdominal wall defects under specific clinical conditions including emergency scenarios is viable. Specialized and experienced groups show lessen surgical site occurrences; further studies are needed to confirm our results.

\section{Background}

Abdominal wall defects are considered a complex pathology with increasing prevalence in the surgical practice [1]. Patients that underwent laparotomy have a $22-55 \%$ risk of herniation [1, 2]. Numerous risk factors have been described in abdominal wall defects, such as emergency procedure, longevity, and comorbidities like chronic pulmonary obstructive disease (COPD), renal impairment, immunosuppression, mal-nutrition, and diabetes mellitus (T2DM) [2]. Frequently, patients that underwent abdominal surgical 
procedures due to critical illness, must endure complex abdominal wall defects that directly impact their quality of life after survival [3]. Additionally, failure of hernia repair comprises between $25-54 \%$ of cases, resulting in an increment of costs, morbidity, and impact on quality of life [4-8]. Therefore, the need for further surgical procedures to correct abdominal wall defects, represents a challenge even for experienced surgeons, seeking a reliable, safe, and durable repair [2-4].

Novitsky et al in 2012, presented a novel technique that modified the traditional surgical approach (Usually Rives-Stoppa procedure) for patients with important abdominal wall defects [5]. As a result, the Transversus abdominis muscle release (TAR) was proposed, showing good results in terms of recurrence, and postoperative and intraoperative complications [5]. TAR technique is based on the principal goals of an abdominal wall reconstruction: restoration of abdominal wall functionality preserving autologous tissue, and reinforcement by a durable mesh with less proportion of complications $[5,6]$. After the presentation of this novel technique, numerous centers started its implementation as part of their regular practice reporting similar results, such as Appleton et al and Pujani et al, finding low incidence of recurrence, and morbidity [1-2].

Surgical expertise is the result of experience associated with the volume of procedures and hours of training, and the impact it may have on postoperative outcomes [9]. Relationship between surgeon volume and postoperative outcomes have been established in different studies in terms of abdominal wall hernia repair, defining low volume as $<12$ hernia repairs, intermediate 12-23 procedures, high 24-35 surgeries, and very high $>36$ procedures [10]. Aquina et al found a positive association between surgeons with intermediate, high, and very high volume and a decrease in hernia recurrence, postoperative complications, and hospital costs [10]. Hence, excellence centers and groups with experienced surgeons have been created through the years with the goal of achieving better results, as presented by Giron et al, this specialization may result in better outcomes [11-14]. Therefore, we present the experience of an abdominal wall reconstruction group (AWRG) outcomes with total abdominis release procedure for complex ventral hernias.

\section{Methods}

\section{Study population}

With the Institutional (Hospital Universitario Mayor Méderi) Review Board's approval and following Health Insurance Portability and Accountability Act (HIPAA) guidelines, a retrospective review of a prospectively collected database was conducted. All patients over 18 years of age that underwent TAR procedure between January 2014 and December 2020 were included. Patients with no surgical description and missing data were excluded. Ethical compliance with the Helsinki Declaration, current legislation on research Res. 008430 - 1993 and Res. 2378 - 2008 (Colombia) and the International Committee of Medical Journal Editors (ICMJE) were ensured under our Ethics and Research Institutional Committee (IRB) approval.

\section{Follow up}


Preoperative data included patient demographics, comorbidities, surgical history, CeDAR score and CT results. Intraoperative and postoperative data included surgical findings, type of mesh, type of mesh fixation, 30 days morbidity and mortality, and follow up. In-hospital postoperative complications were assessed by the same surgeons included in the AWRG. Follow up data at 8 days, 1, 12, 30, and 60 months was acquired, when possible, regardless of the hernia size. All patients were appointed at the same time in external evaluation. A new physical exam of all patients was performed when possible. In the follow up meeting with the patient, a quality life survey (SF-36) was conducted, alongside physical exam, and anamnesis to evaluate postoperative evolution, and hernia recurrence.

\section{Statistical analysis}

Descriptive statistics were reported in terms of variable nature. Qualitative analysis was performed in terms of frequencies and percentages, while quantitative analysis was done in terms of mean and standard deviations of normally distributed data and medians and interquartile ranges (IQRs) for nonnormally distributed data. Bivariate analysis was performed. Qualitative variables were analyzed using chi-square statistics (Fisher's exact test when appropriate). Quantitative variables were analyzed, based on normality, with Spearman's or Pearson's associations correlation coefficients accordingly. Bivariate analysis between qualitative and quantitative variables was performed using Mann-Whitney test or the ttest for independent samples. For associations between categorical variables, odds ratios with $95 \%$ confidence intervals were provided. Multivariate analysis was performed, including all variables that showed association with significant statistical value of $p<0.2$ based on bivariate analysis, also, multivariate results were analyzed with $p<0.05$ cut off point.

\section{Surgical Technique}

Midline incision is performed, based on preoperative CT assessment, to avoid inadvertent enterotomy. In midline defects, dissection of the Hernial sac is performed to free it from adjacent soft tissue. Lysis of parietal adhesions is accomplished using blunt and sharp dissection. Abdominal cavity is packed with warmed compresses for visceral protection. Posterior rectus sheath is divided, on one side, lateral to linea alba. Blunt dissection (hydrodissection) of adhesions is performed until lateral neurovascular bundles are visualized medial to semilunar line. On the other side, superficial pass of the hernial sac allows access to retro rectus space. Retro rectus dissection is performed as on the previous side. Falciform is dissected of línea alba and posterior rectus sheath (PRS). Medial edges of PRS are divided (extra-peritoneally) until rostral ends at the xiphoid within the fatty triangle of Schumpelick [2]. Preperitoneal dissection is performed inferiorly to the arcuate line (Retzius space) as in classic Rives-Stoppa dissection. At epigastrium, the muscular belly is exposed by turning the musculoaponeurotic junction of Transversus Abdominis (TA) medially. TAR begins by dividing the posterior lamina of internal oblique, revealing TA's fibers, which are divided using monopolar energy. The pre-transversalis plane is dissected bluntly until psoas is visualized. TA division is extended medially inte epigastrium and parallel to the musculoaponeurotic line of transition connecting to the opposite side. Reconstruction of the visceral sac is accomplished by suturing the PRS-peritoneum complex with absorbable sutures (delayed). Large polypropylene mesh (synthetic absorbable or composite mesh was used if posterior fascia was deficient) 
is placed extra peritoneally, anchoring one corner to the Copper's ligament. Subfascial suction drains were placed in difficult dissection cases. Anterior sheath is closed with polydioxanone 0 running suture. " $U$ " shaped sutures were placed in places where appreciable tensión was identified during closure. Redundant subcutaneous tissue and lean skin flaps were excised.

\section{Abdominal Wall Reconstruction Group (AWRG) and preoperative assessment}

Since 2014, in our institution, experienced surgeons (5 out of 25 staff surgeons) with interest in open and laparoscopic procedures created a group specialized in abdominal wall pathology and reconstruction (AWRG). All the patients with abdominal wall defect (inguinal, ventral, lateral) were assessed in a multidisciplinary board including surgery, anesthesia, and nutritional support. Patient's selection process was based on institutional guidelines. Measurement of hernia defect was performed based on tomography findings. Surgical risk was calculated using preoperative scores (CeDAR and NSQIP risk score of the ACS). Risk of wound infection was assessed based on past clinical history (malignancy, smoking, corticosteroids, and chemotherapy). Smoking habit was stopped 30 days prior to surgery for all patients. After surgical board review, the type of procedure and mesh placement was defined and explained to the patient.

\section{Results}

Preoperative characteristics. A total of 50 patients underwent TAR procedure for correction of complex abdominal wall defects. $62 \%$ of patients were male. Mean age was $55 \pm 13.4$ years. Mean BMI was 27.8 $\pm 4.5 \mathrm{Kg} / \mathrm{m} 2$. History of hypertension was presented in $34 \%$, T2DM in 7 patients, history of smoking in $34 \%$ of all patients and $92 \%$ had a surgical history with median laparotomy incision (Table $1-$ See appendix). In most of the cases surgical procedure was performed by AWRG surgeons (94\%). Comparison between AWRG vs non AWRG are described in table 7 (Table 7 - See appendix).

Mean gap size of abdominal wall defects was $11.49 \pm 4.03$, with $72 \%$ of all cases classified as W3 (EHS classification) defects. $4 \%$ of patients had a W1 (EHS classification) defect but all of them were recurrent hernias. $28 \%$ of cases were W1 or W2 but had concomitant lateral hernias. TAR procedure was performed in an emergency context in $8 \%$ of all cases. Patients that underwent TAR procedure under emergency conditions, have $0 \%$ rate of re-interventions, and $12,5 \%$ of seroma and superficial surgical site infection. Preoperative pneumoperitoneum was indicated in 9 patients, with a mean amount of $1383 \mathrm{cc}$ and maximum of $5000 \mathrm{cc}$. Preoperative risk was analyzed by the surgical board using CeDAR score with a mean of $20.5 \pm 14.5$. (Table $2-$ See appendix)

Intraoperative characteristics. Surgical mesh was used in all cases using lightweight polypropylene in $90 \%$ of the patients, and in $100 \%$ of the cases we use a bare mesh. Discontinuous suture was the preferred fixation method (68\%), self-fixation mesh was used only in $2 \%$ of all patients. Mean intraoperative bleeding was $101 \mathrm{cc} \pm 112.5$, with mean surgical time of $225 \mathrm{~min} \pm 59.9 \mathrm{~min}$. Additional 
procedures were performed in $20 \%$ of cases (such as liberation of adhesions or umbilical hernia repair) (Table 3 - See appendix)

Complications and follow up. $2 \%$ of patients had hematoma, 4 patients presented seroma. Surgical site infection was divided into superficial (10\%), deep (4\%), and organ-related $(2 \%)$. Pulmonary thromboembolism was presented in $6 \%$ of cases. Only 5 patients required surgical re-intervention: 3 patients require surgical wound debridation, and 2 patients required surgical drainage of postoperative hematoma. None of our patients require mesh explant, or open abdomen. In terms of hernia relapse, we have $12 \%$ of cases; all of them over a year after the surgery. In our center, $94 \%$ of TAR procedures were performed by the AWRG. (Table 4- See appendix)

\section{Hernia recurrence}

In bivariate analysis diabetes mellitus (T2DM) OR $0.75(0.5-0.9)(p=0.131)$, smoking habit OR 0.55 $(0.3-0.7)(p=0.05)$, and history of recurrent hernia OR $0.66(0.2-0.8)(p=0.122)$ were selected for multivariate analysis. Nonetheless, there was no statistically significant association found in the multivariate analysis.

\section{Hematoma, Seroma, Surgical site infection (SSI) and reintervention.}

In multivariate analysis, associated factors for hematoma in postoperative follow up were smoking OR $0.49(0.2-0.63)(p=0.02)$, use of preoperative Botox OR $1.0(0.3-1.1)(p=0.00)$, and pneumoperitoneum OR $0.7(0.3-0.89)(p=0.05)$. In terms of seroma, emergency context OR $0.9(0.5-1.1)(p=0.2)$, and surgery performed by the AWRG OR $1.0(0.8-1.2)(p=0.2)$ were included in multivariate analysis, but none of them showed significant statistical value.

Associated factors for surgical site infection, in multivariate analysis, were diabetes mellitus OR 1.1 $(0.66-1.35)(p=0.01)$, COPD OR $0.5(0.25-0.65)(p=0.08)$, surgery performed by the AWRG OR 0.07 $(0.05-0.1)(p=0.009)$ and surgery performed by surgeons outside specialized group OR $13.6(9.5-15.1)$ $(p=0.04)$. In terms of surgical reintervention, in multivariate analysis, the only associated factor was chronic renal impairment OR $1.5(0.9-1.2)(p=0.001)$. (Table 5 - See appendix))

\section{Discussion}

Throughout time, complex abdominal wall defects have increased due to patients' possibility to overcome critical illness after laparotomy [15], presenting a new surgical challenge that requires a wide armamentarium of surgical techniques and approaches, seeking to provide a proper reconstruction, assuring functionality of the abdominal wall [16]. Several advances in hernia surgery have been presented to achieve durable and safe repairs, trying to gain muscular tissue [16]. Initially, Ramirez et al described an anterior component separation technique, nonetheless, due to the high proportion of morbidity associated with large skin flaps, other techniques were proposed. Therefore, retro muscular techniques were developed based on River-Stoppa procedure, showing a safe, and durable repair despite 
high risk of neurovascular bundle damage $[16,17]$. Novitsky et al, to improve retro muscular repair, proposed a posterior component separation with transversus abdominis release procedure, showing positive results, with less proportion of recurrence ( $<4 \%$ in 12 months), postoperative complications (hematoma ( $<1 \%)$, seroma ( $<3 \%)$, surgical site infection (SSI) $(<10 \%)$ ) and an acceptable median length of hospitalization of 5.9 days $[4,16]$.

Several risk factors have been described for postoperative complications, and recurrence rates [18]. Factors associated with higher recurrence rates described in literature are obesity (BMI greater than 25 $\mathrm{kg} / \mathrm{m} 2$ ), smoking history, T2DM, corticosteroid use, and procedure performed in emergency context [1921]. In terms of postoperative complications such as seroma, hematoma and SSI, a clear association has been determined with smoking history, T2DM, and COPD [22-25]. In this study, smoking history OR 0.49 (0.2-0.63) ( $p=0.02)$, use of Botox OR $1.0(0.3-1.1)(P=0.00)$, and pneumoperitoneum OR $0.7(0.3-0.89)$ $(p=0.05)$ have shown association with presence of hematoma, in accordance to Lindstrom et al where a clear relationship of tobacco consumption and postoperative complications was addressed [24-32]. Fields et al, Hellspong et al and Martin et al Identified T2DM and COPD as risk factors for SSI, like the association found in our study (T2DM OR $1.1(0.66-1.35)(p=0.01)$, COPD OR $0.5(0.25-0.65)(p=0.08)$ $[24,25,33]$. In terms of reintervention, Chronic renal impairment showed a positive association OR 1.5 $(0.9-1.2)(p=0.001)$, probably related to renal wound healing disturbances described in literature [34] .

Due to the complexity in the management of complex abdominal wall defects, creation of surgical groups specialized has arisen as a feasible option seeking achievement of better postoperative outcomes, such as morbidity, mortality, and recurrence rate reduction in these patients. Chatta et al and Raigani et al analyzed the impact in terms of surgical outcomes and financial burden, showing that patients who underwent treatment in high volume specialized center have lower rate of overall complications ( $9.5 \%$ vs $8 \%), \mathrm{SSI}(6.2$ vs $5 \%)$ and reduced in hospital stay, with an increased financial burden $(\mathrm{OR}=1.21)[26,27]$.

Mean defect gap size was $11.40 \mathrm{~cm} \pm 4.03$, with a median operative time and mean intraoperative bleeding of $275 \mathrm{~min}$ and $101 \mathrm{ml}$, like results described in literature [4, 16]. Postoperative complication rates may vary from 0.5 to $17 \%$. Novitsky et al reported 428 patients that underwent TAR procedure, $9.1 \%$ had SSI (Superficial 78\%, Deep 18\%), 2.8\% seroma, 0.8\% hematoma and 0.8\% granuloma [16], results like those found in our population with a slightly higher presence of seroma, but less presence of deep SSI and no cases of granuloma. (Superficial SSI 10\%, deep SSI 4\%, Hematoma 1\%, Seroma $8 \%$ ). Other complications were documented (Thromboembolic events $6.3 \%$ and pneumonia $1.4 \%$ ), with no difference with data reported in other studies $[10,16]$.

Recurrence rate is considered a cornerstone in hernia surgery follow up, leading to the invention of new methods of mesh fixation to reduce its presentation (Traditional fixation methods, self-gripping mesh, biological adhesives, fibrin glue) [28, 29]; nonetheless, in our population, $94 \%$ of the meshes were fixated with absorbing monofilament sutures and only in $2 \%$ of cases self-fixation mesh was used. Wheeler et al and Mehrabi et al described a recurrence rate, in retro muscular repair technique (River-Stoppa), from 5$7.3 \%$ [30-31], Novitsky et al reported a recurrence rate of $3.7 \%$ with TAR procedure [16], being these 
results akin to those found in our population, where recurrence rate at follow up was $12 \%$, with a mean follow up of 35,72 months ( 12 month $=4 \%$ ). Surgeon expertise and high/intermediate volume centers are related with better postoperative outcomes, and lees financial burden in hernia surgery (9-14); in our institution, a protective factor in terms of surgical site infection, with a OR 0.07 IC $(p=0,2)$ was found if patients underwent TAR procedure performed by AWRG, conversely to a 13-fold risk increase in SSI if the procedure was not performed by AWRG. Patients that underwent TAR procedure performed by AWRG show $8 \%$ of hernia relapse, compared with non AWRG surgeons with $0 \%$ of relapse. These could be explained by the high complexity procedures performed by surgeons included in the abdominal wall reconstruction group.

In our study, almost $8 \%$ of the patients underwent TAR procedure in an emergency context; according to Alkhatib et al [34], there is a $13,6 \%$ of patients that could require re-interventions due to wound complications such as : surgical site infection (13,6\%), and $25,6 \%$ total reports of wound occurrences; this data is comparable with our results, in the population analyzed in our study, there are no re-interventions and $12,5 \%$ of wound complications (seroma and superficial surgical site infection); however all the emergency procedures were performed by surgeons included in the abdominal wall reconstruction group.

Abdominal wall defect procedures are designed aiming to not only repair the defect but also to restore the functionality of the abdominal wall, influencing patient's self-esteem, emotional and mental health, being life quality an important quality indicator in hernia surgery [35-37]. In our study, life quality evaluation showed great results with 93.8 pts in change of health, 88.5 in social function, 83.8 in role limitation (Table 6 - See appendix), measured by the -ShortForm36- [38]. Based on these results we can assert that a multidisciplinary and specialized group can offer better postoperative outcomes, reduce in-hospital costs, and have a positive impact on the life quality of our patients with complex abdominal wall defects.

Among the limitations of this study are its retrospective nature and the lack of previous studies to compare our results in terms of abdominal group versus non-specialized surgical team. Although studies have appeared in recent years regarding this topic, further prospective studies are needed to validate our results.

\section{Conclusion}

Transverse Abdominis Release (TAR) procedure for complex abdominal wall defects under specific clinical conditions including emergency scenarios is viable. Experienced groups show better postoperative outcomes and lessen surgical site infection with great results in terms of life quality according to our results, however further prospective studies are needed to confirm our results.

\section{Declarations}

Data Availability: The datasets generated and/or analysed during the current study are not publicly available due to institutional data protection but are available from the corresponding author on 
reasonable request

\section{Compliance with Ethical Standards:}

Funding: This study does not receive any funding.

Conflict of Interest: Authors do not have any conflict of interest.

Ethical approval: All procedures performed in studies involving human participants were in accordance with the ethical standards of the institutional and/or national research committee and with the 1964 Helsinki declaration and its later amendments or comparable ethical standards.

Informed consent: Informed consent was obtained from all individual participants included in the study, and reposes in clinical history

\section{References}

1. Punjani R, Arora E, Mankeshwar R, Gala J. An early experience with transversus abdominis release for complex ventral hernias: a retrospective review of 100 cases. Hernia. 2021 Apr;25(2):353-364. doi: 10.1007/s10029-020-02202-w. Epub 2020 May 6. PMID: 32377962

2. Appleton ND, Anderson KD, Hancock K, Scott MH, Walsh CJ. Initial UK experience with transversus abdominis muscle release for posterior components separation in abdominal wall reconstruction of large or complex ventral hernias: a combined approach by general and plastic surgeons. Ann R Coll Surg Engl. 2017 Apr;99(4):265-270. doi: 10.1308/rcsann.2016.0241. Epub 2016 Aug 11. PMID: 27513800; PMCID: PMC5449665

3. By: Zarzaur, B.L.; Dicocco, J.M.; Shahan, C.P.; Emmett, K.; Magnotti, L.J.; Croce, M.A.; Fabian, T.C.; Hathaway, D.K.. Journal of Trauma - Injury, Infection and Critical Care, February 2011, 70(2):285-291 Language: English. DOI: 10.1097/TA.0b013e31820b5b80

4. Novitsky YW, Elliott HL, Orenstein SB, Rosen MJ. Transversus abdominis muscle release: a novel approach to posterior component separation during complex abdominal wall reconstruction. Am J Surg. 2012 Nov;204(5):709-16. doi: 10.1016/j.amjsurg.2012.02.008. Epub 2012 May 16. PMID: 22607741

5. Reynolds D, Davenport DL, Korosec RL, Roth JS. Financial implications of ventral hernia repair: a hospital cost analysis. J Gastrointest Surg. 2013 Jan;17(1):159 - 66; discussion p.166-7. doi: 
10.1007/s11605-012-1999-y. Epub 2012 Sep 11. PMID: 22965649.

6. Breuing K., Butler C.E., Ferzoco S., et. al.: Incisional ventral hernias: review of the literature and recommendations regarding the grading and technique of repair. Surgery 2010; 148: pp. 544-558

7. .Grove, T.N., Muirhead, L.J., Parker, S.G. et al. Measuring quality of life in patients with abdominal wall hernias: a systematic review of available tools. Hernia 25, 491-500 (2021).

https://doi.org/10.1007/s10029-020-02210-w

8. .Blair LJ, Cox TC, Huntington CR, Groene SA, Prasad T, Lincourt AE, Kercher KW, Heniford BT, Augenstein VA. The effect of component separation technique on quality of life (QOL) and surgical outcomes in complex open ventral hernia repair (OVHR). Surg Endosc. 2017 Sep;31(9):3539-3546. doi: 10.1007/s00464-016-5382-z. Epub 2016 Dec 30. PMID: 28039655.

9. Neumayer LA, Gawande AA, Wang J, Giobbie-Hurder A, Itani KM, Fitzgibbons RJ Jr, Reda D, Jonasson $0 ;$ CSP \#456 Investigators. Proficiency of surgeons in inguinal hernia repair: effect of experience and age. Ann Surg. 2005 Sep;242(3):344-8; discussion 348 - 52. doi:

10.1097/01.sla.0000179644.02187.ea. PMID: 16135920; PMCID: PMC1357742.

10. Aquina, C.T., Kelly, K.N., Probst, C.P. et al. Surgeon Volume Plays a Significant Role in Outcomes and Cost Following Open Incisional Hernia Repair. J Gastrointest Surg 19, 100-110 (2015). https://doiorg.ez.urosario.edu.co/10.1007/s11605-014-2627-9

11. Schlosser KA, Arnold MR, Kao AM, Augenstein VA, Heniford BT. Building a Multidisciplinary HospitalBased Abdominal Wall Reconstruction Program: Nuts and Bolts. Plast Reconstr Surg. 2018 Sep;142(3 Suppl):201S-208S. doi: 10.1097/PRS.0000000000004879. PMID: 30138291.

12. Hawn MT, Snyder CW, Graham LA, Gray SH, Finan KR, Vick CC. Hospital-level variability in incisional hernia repair technique affects patient outcomes. Surgery 2011;149:185-91.

13. Borenstein SH, To T, Wajja A, Langer JC. Effect of subspecialty training and volume on outcome after pediatric inguinal hernia repair. Journal of pediatric surgery 2005;40:75-80.

14. Girón, F., Hernandez, J.D., Linares, J.D. et al. Outcomes of 207 totally extraperitoneal hernia repairs using self-fixation mesh. Sci Rep 11, 12507 (2021). https://doi.org/10.1038/s41598-021-92063-9

15. Amjad Shah, Zia Aftab, Syed Muhammad Ali, Salah Gehani, Khalid Ahmed, Ahmed Almodaris, Rashad Fouad, Abdulrahman Al-Aal, Incisional Hernia Post Laparotomy-Incidence and Risk Factors, Journal of Surgery. Vol. 6, No. 1, 2018, pp. 19-22. doi: 10.11648/j.js.20180601.14

16. Novitsky, Y., Fayezizadeh, M., Majumder, A., Neupane, R., Elliott, H., Orenstein, S. \& (2016). Outcomes of Posterior Component Separation With Transversus Abdominis Muscle Release and Synthetic Mesh Sublay Reinforcement. Annals of Surgery, 264 (2), 226-232. doi: 10.1097/SLA.0000000000001673.

17. Ramirez OM, Ruas E, Dellon AL. Components separation" method forclosure of abdominal-wall defects: an anatomic and clinical study. Plast Reconstr Surg1990;86:519-526.

18. Lindmark, M., Strigård, K., Löwenmark, T. et al. Risk Factors for Surgical Complications in Ventral Hernia Repair. World J Surg 42, 3528-3536 (2018). https://doi.org/10.1007/s00268-018-4642-6 
19. S G Parker, S. Mallett, L Quinn, C P J Wood, R W Boulton, S Jamshaid, M. Erotocritou, S. Gowda, W. Collier, A A O Plumb, A C J Windsor, L Archer, S Halligan, Identifying predictors of ventral hernia recurrence: systematic review and meta-analysis, BJS Open, Volume 5, Issue 2, March 2021, zraa071, https://doi.org/10.1093/bjsopen/zraa071

20. Sauerland S, Korenkov M, Kleinen T, Arndt M, Paul A. Obesity is a risk factor for recurrence after incisional hernia repair. Hernia. 2004 Feb;8(1):42-6. doi: 10.1007/s10029-003-0161-x. Epub 2003 Sep 6. PMID: 13680307.

21. Wink JD, Wes AM, Fischer JP, Nelson JA, Stranksy C, Kovach SJ 3rd. Risk factors associated with early failure in complex abdominal wall reconstruction: a 5 year single surgeon experience. J Plast Surg Hand Surg. 2015 Apr;49(2):77-82. doi: 10.3109/2000656X.2014.903195. Epub 2014 Apr 3. PMID: 24693869.

22. Landin M, Kubasiak JC, Schimpke S, Poirier J, Myers JA, Millikan KW, Luu MB. The effect of tobacco use on outcomes of laparoscopic and open inguinal hernia repairs: a review of the NSQIP dataset. Surg Endosc. 2017 Feb;31(2):917-921. doi: 10.1007/s00464-016-5055-y. Epub 2016 Jun 28. PMID: 27351659.

23. Mercoli H, Tzedakis S, D'Urso A, Nedelcu M, Memeo R, Meyer N, Vix M, Perretta S, Mutter D. Postoperative complications as an independent risk factor for recurrence after laparoscopic ventral hernia repair: a prospective study of 417 patients with long-term follow-up. Surg Endosc. 2017 Mar;31(3):1469-1477. doi: 10.1007/s00464-016-5140-2. Epub 2016 Aug 5. PMID: 27495336.

24. Fields AC, Divino CM. Surgical outcomes in patients with chronic obstructive pulmonary disease undergoing abdominal operations: An analysis of 331,425 patients. Surgery. 2016 Apr;159(4):12106. doi: 10.1016/j.surg.2015.11.007. Epub 2015 Dec 15. PMID: 26704782.

25. Hellspong G, Gunnarsson U, Dahlstrand U, Sandblom G. Diabetes as a risk factor in patients undergoing groin hernia surgery. Langenbecks Arch Surg. 2017 Mar;402(2):219-225. doi: 10.1007/s00423-016-1519-8. Epub 2016 Dec 7. PMID: 27928640.

26. Chattha A, Muste J, Patel A. The impact of hospital volume on clinical and economic outcomes in ventral hernia repair: an analysis with national policy implications. Hernia. 2018 Oct;22(5):793-799. doi: 10.1007/s10029-018-1803-3. Epub 2018 Aug 9. PMID: 30094752

27. Raigani S, De Silva GS, Criss CN, Novitsky YW, Rosen MJ. The impact of developing a comprehensive hernia center on the referral patterns and complexity of hernia care. Hernia. 2014 0ct;18(5):625-30. doi: 10.1007/s10029-014-1279-8. Epub 2014 Jul 20. PMID: 25038895.

28. Mitura K, Garnysz K, Michałek I. Long-term follow-up of a randomized controlled trial of Lichtenstein repair vs the Valenti technique for inguinal hernia. Hernia. 2019 Jun;23(3):547-554. doi: 10.1007/s10029-019-01879-y. Epub 2019 Jan 18. PMID: 30659397; PMCID: PMC6586697.

29. Ozmen J, Choi V, Hepburn K, Hawkins W, Loi K. Laparoscopic Totally Extraperitoneal Groin Hernia Repair Using a Self-Gripping Mesh: Clinical Results of 235 Primary and Recurrent Groin Hernias. J Laparoendosc Adv Surg Tech A. 2015 Nov;25(11):915-9. doi: 10.1089/lap.2015.0056. Epub 2015 Nov 2. PMID: 26523915. 
30. Wheeler AA, Matz ST, Bachman SL, et al. Retrorectus polyester mesh repair for midline ventral hernias. Hernia. 2009;13:597-603.

31. Mehrabi M, Jangjoo A, Tavoosi $H$, et al. Long-term outcome of Rives-Stoppa technique in complex ventral incisional hernia repair. World J Surg. 2010;34:1696-1701.

32. Lindström D, Sadr Azodi O, Bellocco R, Wladis A, Linder S, Adami J. The effect of tobacco consumption and body mass index on complications and hospital stay after inguinal hernia surgery. Hernia. 2007 Apr;11(2):117-23. doi: 10.1007/s10029-006-0173-4. Epub 2006 Dec 6. PMID: 17149530.

33. Martin ET, Kaye KS, Knott C, Nguyen H, Santarossa M, Evans R, Bertran E, Jaber L. Diabetes and Risk of Surgical Site Infection: A Systematic Review and Meta-analysis. Infect Control Hosp Epidemiol. 2016 Jan;37(1):88-99. doi: 10.1017/ice.2015.249. Epub 2015 Oct 27. PMID: 26503187; PMCID: PMC4914132.

34. Alkhatib H, Tastaldi L, Krpata DM, Petro CC, Olson M, Rosenblatt S, Rosen MJ, Prabhu AS. Outcomes of transversus abdominis release in non-elective incisional hernia repair: a retrospective review of the Americas Hernia Society Quality Collaborative (AHSQC). Hernia. 2019 Feb;23(1):43-49. doi: 10.1007/s10029-019-01878-z. Epub 2019 Jan 9. PMID: 30627813.

35. Heller A, Westphal SE, Bartsch P, Haase M, Mertens PR. Chronic kidney disease is associated with high abdominal incisional hernia rates and wound healing disturbances. Int Urol Nephrol. 2014 Jun;46(6):1175-81. doi: 10.1007/s11255-013-0565-1. PMID: 24078009.

36. Horzić M, Kopljar M, Cupurdija K, Skupnjak M, Korica J, Lacković Z, Vanjak-Bielen D, Patrlj L. Quality of life changes after inguinal hernia repair using anterior rectus sheath-a preliminary study. Coll Antropol. 2006 Jun;30(2):349-53. PMID: 16848150.

37. Jensen KK, Emmertsen KJ, Laurberg S, Krarup PM. Long-term impact of incisional hernia on quality of life after colonic cancer resection. Hernia. 2020 Apr;24(2):265-272. doi: 10.1007/s10029-01901978-w. Epub 2019 May 24. PMID: 31127401.

38. Jensen KK, Henriksen NA, Harling H. Standardized measurement of quality of life after incisional hernia repair: a systematic review. Am J Surg. 2014 Sep;208(3):485-93. doi: 10.1016/j.amjsurg.2014.04.004. Epub 2014 Jun 12. PMID: 25017051.

39. Lawrence K, McWhinnie D, Jenkinson C, Coulter A. Quality of life in patients undergoing inguinal hernia repair. Ann R Coll Surg Engl. 1997 Jan;79(1):40-5. PMID: 9038494; PMCID: PMC2502599.

\section{Supplementary Files}

This is a list of supplementary files associated with this preprint. Click to download.

- AppendixTAR.docx

- Datatar.docx 